\title{
Minimum Period of Rotation of Millisecond Pulsars and Pulsar Matter Equations of State
}

\author{
Sergey Mikheev ${ }^{1, \star}$ and Victor Tsvetkov ${ }^{1, \star \star}$ \\ ${ }^{1}$ Tver State University
}

\begin{abstract}
Based on the findings of our previous studies of fast-rotating Newtonian polytropes, we found the relation between the minimum pulsar rotation period, the value of pulsar central density, and the polytropic index. From this relation we come to the conclusion that the value of minimum central density of a pulsar with a peak period is $2.5088 \cdot 10^{14} \mathrm{~g} / \mathrm{cm}^{3}$.
\end{abstract}

\section{Introduction}

The astrophysical data is indicative of the presence of a minimum period of rotation of the millisecond pulsars. Now this period is $1.396648 \mathrm{~ms}$. The indicated value is reached long before the pulsar destruction under the influence of centrifugal forces. This phenomenon is easily explained by the fast growth of the angular momentum losses near the bifurcation point of the pulsar configuration due to intense pulsar gravitational radiation.

On the 11th of February 2016 the experimental discovery of gravitational waves by collaborations LIGO and VIRGO was announced [1].

We suggest the possibility of detection of gravitational waves generated by rapidly rotating magnetized gravitating configurations (pulsars) [2].

\section{The Polytrope Equation of State of a Pulsar}

For designation of gravitating configurations with the equations of state for matter in the form of polytropes the term Newtonian polytrope is generally accepted [3].

As shown in [4], both the ideal Fermi gas equation of state and the realistic Bethe-Johnson and Raid equations can be approximated by the polytrope with the corresponding index $n$.

Assume that the pulsar configuration density is given by:

$$
\rho=\sum_{a+b+c=0}^{a+b+c=P} \rho_{a b c} x^{a} y^{b} z^{c} .
$$

The parameter $X$ determining the intensity of pulsar gravitational radiation is given by:

$$
X=\rho_{[20] 0}=\rho_{200}-\rho_{020} .
$$

\footnotetext{
${ }^{\star}$ e-mail: Mikheev.SA@tversu.ru
}

$\star \star$ e-mail: Tsvetkov.VP@tversu.ru 
In our papers $[5,6]$ the equation for the definition of the $X$-values was proposed:

$$
A(\varepsilon, n) X+b_{k}(n) X^{3}=\eta_{m},
$$

where $\varepsilon=\omega^{2} /\left(4 \pi G \rho_{0}\right), \omega=2 \pi / T, T$ is the pulsar rotation period, $\rho_{0}$ is the pulsar center density, $\eta_{m}=B_{0 i n}^{2} \sin ^{2} \alpha /\left(32 \pi^{2} G \rho_{0}^{2} a^{2}\right), a$ is the equatorial radius of the configuration, $G$ is the gravitation constant, $B_{0}$ is the effective value of intensity of internal magnetic field at the center of configuration.

According to the mathematical catastrophe theory, the equation (3) describes a classic case of catastrophe $A_{3}$ iff $\eta_{m}=0$.

Let the critical points $\varepsilon_{k}$ be defined by the statement:

$$
A\left(\varepsilon_{k}, n\right)=0 .
$$

Then $\varepsilon_{k}=\varepsilon_{k}(n)$.

At the critical points, $X=X_{k}=\sqrt[3]{\eta_{m} / b_{k}}$, and away from the critical points, $X=\eta_{m} / A(\varepsilon, n)$. For real pulsars, $\eta_{m} \sim 10^{-9}-10^{-12}$. Therefore $X_{k}$ and $X$ can differ by 6-8 orders of magnitude.

The parameter $X$ determines the intensity of pulsar gravitational radiation, and correspondingly at the critical points the intensity will be higher by many orders of magnitude in comparison with its value away from the critical points.

The calculation of $\varepsilon_{k}(n)$ was carried out using a program package developed and implemented by us $[5,6]$.

The $\varepsilon_{k}(n)$ plot is given in Fig. 1.

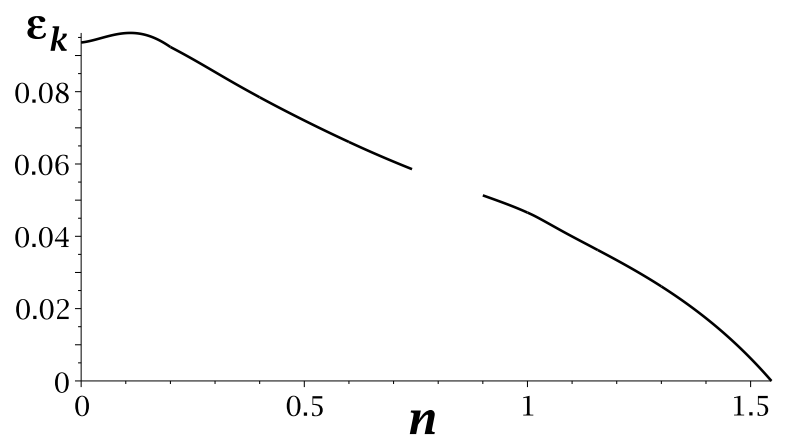

Figure 1. The plot of the function $\varepsilon_{k}(n)$

From Fig. 1 we can see that there are no critical points at polytropic index values $0.74 \leq n \leq 0.904$ and $n \geq 1.55$, and therefore, the gravitational radiation of pulsars with these state equations will be weaker by many orders of magnitude.

The intense gravitational radiation of a pulsar leads to the loss of its angular momentum $M$ according to the following equation:

$$
\dot{M}=-\omega^{-1} I_{g},
$$

where $I_{g}$ is the intensity of its gravitational radiation.

The growth of the rotational speed of a newly born neutron star is responsible for the rapid decrease in its radius at the moment of its birth, and in a dual system - for twisting as well the accretion of matter from the second component. According to the equation (5), the growth of the angular speed of the pulsar rotation at the critical point $\omega_{k}$ will be stopped due to the fast growth of the gravitational 
radiation intensity near this point. Thus, the millisecond pulsar is born with the lowest value of the rotation period. From (5) the following relation arises:

$$
T>T_{k}=\sqrt{\pi /\left(G \rho_{0} \varepsilon_{k}(n)\right)} .
$$

From (6) the function $\rho_{0}=\rho_{0}\left(n, T_{k}\right)$ follows which can be represented graphically in Fig. 2.

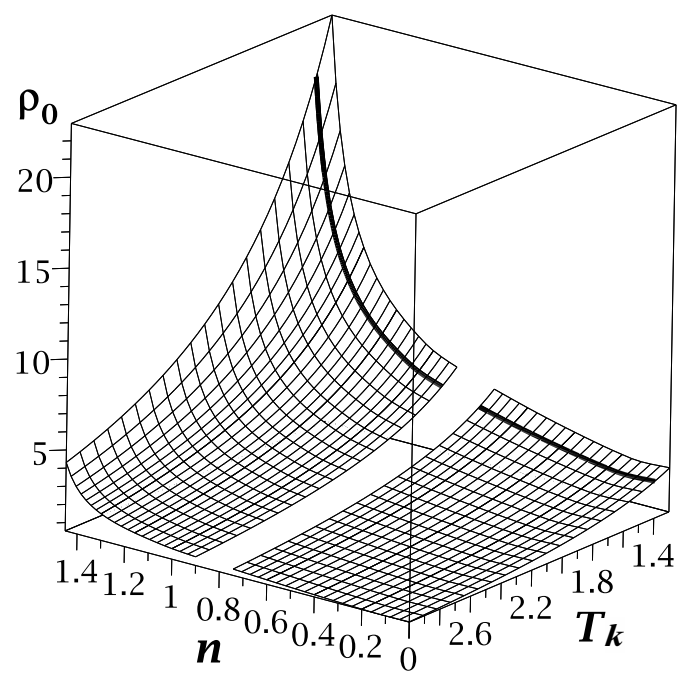

Figure 2. The plot of the function $\rho_{0}\left(n, T_{k}\right)$. The thick line shows the curve $\rho_{0}\left(n, T_{k}=1.396648\right) ; \rho_{0}$ is given in $10^{14} \mathrm{~g} / \mathrm{cm}^{3} ; T_{k}$ is given in $\mathrm{msec}$.

The region of discontinuity of the function $\varepsilon_{k}(n)$ in the interval of $0.74 \leq n \leq 0.904$ leads to a doubly connected surface $\rho_{0}\left(n, T_{k}\right)$ in Fig. 2 . The domain $n \geq 1.55$ does not represent a significant interest for the problem under consideration and is not mapped in Fig. 2.

Fig. 2 displays the presence of ranges of the polytropic index $n$ for which there is no the limitation (6) for the period of the pulsar rotation. At the present time there are all reasons to take the value of $1.396648 \mathrm{~ms}$ for the minimum pulsar rotation period. If pulsars with a substantially smaller period would be found, their equations of state would be close to the polytrope with an index of either $0.74 \leq n \leq 0.904$ or $n \geq 1.55$.

For other polytropic index values, Fig. 2 imposes the constraint: $\rho_{0} \geq 2.5088 \cdot 10^{14} \mathrm{~g} / \mathrm{cm}^{3}$.

Let us consider the possibility of detection of gravitational waves generated by rapidly rotating magnetized gravitating configurations (pulsars) [2].

The possibility of detecting this radiation would allow unique inferences on the equations of state of the pulsar overdense nuclear matter. The pulsar gravitational radiation is generated at doubled 
rotation speed of pulsars and based on the asymmetry parameter of the matter density distribution relative to the X-ray pulsar's rotation axis related to its magnetic field and magnetic axis directed at an angle to the rotation axis.

In principle, in order to obtain any data on object locations, it is necessary to use observation data obtained both by gravitational wave detectors and conventional radiotelescopes. The most precise object direction sensing is provided by its electromagnetic radiation. High hopes follow from the possibility to set up signal match experiments in which gravitational waves will be detected together with electromagnetic ones [2].

First of all, such objects include supernovas which trigger bursts of electromagnetic radiation. If their shapes differ from the axially symmetric one under collapsing, they will also emit gravitational waves. Accordingly, with the help of gravitational waves we can "see" the instant of supernova explosion whereas in the optical channel this information is delayed for several hours required for the explosion development whereafter the instant of supernova explosion can be observed as a stellar flare [2].

If gravitational waves followed by a flare would be detected, this would allow to confirm with a high probability that the detected radiation is really associated with supernova explosion. Besides, such a delay on the path of 50-100 mln light years long would prove that gravitational waves propagate with the speed of light.

\section{Conclusions}

From our report, it follows that the minimum pulsar rotation period of the order of $1.3 \mathrm{~ms}$ observable by experience, indirectly shows that the millisecond pulsar configurations have critical points and there is intense gravitational radiation near these points.

In this paper we have discussed the possibility of detecting gravitational waves generated by rapidly rotating magnetized gravitating configurations (pulsars).

\section{References}

[1] B.P. Abbott et al., Phys. Rev. Let. 116, 061102, 1-16 (2016)

[2] K.S. Torn, Priroda \#4 (860), 34-45 (1987)

[3] S.L. Shapiro and S.A. Teukolsky, Black Holes, White Dwarfs and Neutron Stars: The physics of compact objects (New York: Wiley, 1983), 645 pp.

[4] S.A. Mikheev and V.P. Tsvetkov, Physics of Particles and Nuclei Letters 7, 1, 1-4 (2010)

[5] S.A. Mikheev and V.P. Tsvetkov, Physics of Particles and Nuclei Letters 5, 4, 405-412 (2008)

[6] S.A. Mikheev and V.P. Tsvetkov, Physics of Particles and Nuclei Letters 10, 3, 234-242 (2013) 